\title{
Costos ambulatorios y hospitalarios de la falla cardiaca en dos hospitales de Bogotá
}

\author{
Outpatient and inpatient costs of heart failure in \\ two hospitals in Bogotá
}

\author{
Diana Carolina Tamayo, Viviana Alejandra Rodríguez, María Ximena \\ Rojas, Mónica Rincón, Camilo Franco, María Teresa Ibarra, \\ Rodolfo José Dennis • Bogotá, D.C. (Colombia)
}

\section{Resumen}

Objetivo: realizar una aproximación a la determinación de costos directos de la falla cardiaca (FC) en el país, a través de la evaluación de costos asociados con el cuidado de pacientes atendidos en dos instituciones prestadoras de salud de Bogotá.

Métodos: estudio de costos bajo la perspectiva del tercer pagador. La identificación de eventos generadores de costos en atención ambulatoria se realizó mediante revisión de historias clínicas de pacientes atendidos durante 2011 en la consulta externa especializada de una institución. Los costos de interconsultas y paraclínicos se determinaron según los valores del Acuerdo 256 de 2001, con adición de $30 \%$. Los costos de la medicación se determinaron a partir del registro SISMED. La identificación de eventos generadores de costos en hospitalización se realizó mediante revisión de listados y facturas de pacientes atendidos entre 2009 y 2010 en dos instituciones. Los resultados se presentan resumidos por medidas de tendencia central y de dispersión, en pesos colombianos (COP) de 2011.

Resultados: el costo mensual promedio del tratamiento ambulatorio de FC fue de 304.318 COP (D.E. 760.876), con una mediana de 45.280 COP (RIC 25.539 - 109.715); los medicamentos representaron la fuente principal de consumo de recursos $(55,2 \%)$. El costo promedio de la hospitalización por descompensación de FC fue de 6.427.887 COP (D.E. 9.663.176); la estancia hospitalaria representó la mayor proporción del costo $(29,1 \%)$.

Conclusiones: los costos ambulatorios, y especialmente los hospitalarios, asociados con la FC en Colombia son sustanciales. La fuente principal de costos difiere dependiendo de si el manejo es hospitalario (estancia) o ambulatorio (medicamentos). (Acta Med Colomb 2013; 38: xx-xx).

Palabras clave: falla cardiaca, costos del cuidado de la salud, costos de enfermedad, Colombia.

\section{Abstract}

Objective: to make an approach to the determination of direct costs of heart failure (HF) in the country through the evaluation of costs associated with the care of patients seen in two health institutions in Bogota.

Methods: low cost third-party payer perspective. Identification of cost generating events in ambulatory care was performed by review of medical records of patients seen during 2011 in the specialized outpatient clinic of an institution. Interconsultations and paraclinical costs were determined according to the 256 Agreement of 2001, with addition of $30 \%$. Medication costs were determined from the SISMED register. Identification of events that generate costs in hospitalization was conducted by reviewing lists and bills of patients treated between 2009 and 2010 in two institutions. The results are presented summarized by measures of central tendency and dispersion, in Colombian pesos (COP) of 2011.

Results: the average monthly cost for outpatient treatment of HF was 304,318 COP (D.E. 760 876), with a median of 45,280 COP (RIC 25,539-109,715); drugs represented the main source of resource consumption (55.2\%). The average cost of hospitalization for decompensated HF was 6,427,887 COP (D.E. 9.663.176); hospital stay accounted for the largest proportion of the cost (29.1\%).

Conclusions: outpatient costs, and especially the inpatient ones associated with HF in Colombia are substantial. The main source of costs differs depending on whether the management is hospitable (stay) or outpatient (drugs). (Acta Med Colomb 2013; 38: xx-xx).

Keywords: heart failure, costs of health care, cost of illness, Colombia.

Dra. Diana Carolina Tamayo Fuquen: MSc(c), Departamento de Investigaciones Fundación Cardioinfantil - Instituto de Cardiología, Candidata a Magíster en Epidemiología Clínica, Departamento de Epidemiología Clínica y Bioestadística Pontificia Universidad Javeriana; Dra. Viviana Alejandra Rodríguez: MSc.Departamento de Epidemiología Clínica y Bioestadística, Pontificia Universidad Javeriana; Dra. María Ximena Rojas Reyes: RN, MSc., Departamento de Epidemiología Clínica y Bioestadística, Pontificia Universidad Javeriana; Dra. Mónica Rincón Roncancio: MA, MSc, Magíster en Bioética y Fisiología Fundación Cardioinfantil, Instituto de Cardiología; Dr. Camilo Franco: Jefe de la Clínica de Falla Cardiaca, Fundación Cardioinfantil, Instituto de Cardiología; María Teresa Ibarra: RN, Departamento de Enfermería, Fundación Cardioinfantil, Instituto de Cardiología; Dr. Rodolfo José Dennis Verano: MSc, FACP., Departamento de Investigaciones, Fundación Cardioinfantil, Instituto de Cardiología, Departamento de Epidemiología Clínica y Bioestadística, Pontificia Universidad Javeriana. Bogotá, D.C. (Colombia).

Correspondencia. Dr. Rodolfo José Dennis Verano. Departamento de Investigaciones, Fundación Cardioinfantil, Bogotá, D.C. (Colombia)

E-mail: rdennis@cardioinfantil.org Recibido: 20/III/2013 Aceptado: 28/VI/2013 


\section{Introducción}

La falla cardiaca (FC) es uno de los síndromes cardiovasculares con mayor frecuencia y mejor caracterizados en la literatura. De acuerdo con las estadísticas derivadas del seguimiento de la cohorte de Framingham, la incidencia de FC se aproxima a 10 por cada 10 mil personas mayores de 65 años y se duplica con cada década de la vida; adicionalmente, la hipertensión arterial se identificó como el factor de riesgo más frecuente (antecedente en $75 \%$ de los casos) $(1,2)$. La prevalencia de FC registrada en la literatura internacional se acerca a $2 \%$ en población general y llega hasta $10 \%$ entre los adultos mayores (1).

Caracterizaciones entre población norteamericana reportan una prevalencia de FC de 2,3\% en población general y se considera responsable de aproximadamente un millón de hospitalizaciones al año. Constituye la causa de hospitalización más frecuente en individuos mayores de 65 años. Representó una carga económica calculada en 25 billones de dólares anuales en 2003, cifra que llegó a los 33 ¿illones de dólares en 2007; 75\% de este gasto en FC se annbuyó a los eventos hospitalarios $(1,3)$.

En Latinoamérica, la prevalencia de $\mathrm{FC}$ ha mostrado un incremento debido al aumento en la supervivencia de pacientes con enfermedad isquémica cardiaca y en la expectativa de vida que supera actualmente los 70 años. Además de la hipertensión arterial, cuya prevalencia en Latinoamérica se estima en $18 \%$, en la región se suman otros factores de riesgo como la enfermedad cardiaca reumática y la enfermedad de Chagas, principal causa de discapacidad secundaria a enfermedades tropicales en adultos jóvenes de la región. En Colombia, por ejemplo, $18 \%$ de la población vive en áreas endémicas de la enfermedad de Chagas y se estima que 1,3 millones de personas están infectadas. La edad promedio de los pacientes con FC en Latinoamérica se ha reportado entre 46 y 65 años, inferior a lo descrito en países desarrollados, y con etiologías diversas: enfermedad coronaria entre 17 y $38 \%$ de los casos, hipertensión arterial entre 14 y $32 \%$, enfermedad de Chagas entre 6 y $20 \%$, entre otras $(2,4)$.

Mientras la literatura internacional presenta múltiples estadísticas relacionadas con la epidemiología de la FC, es limitada la publicación de este perfil en Colombia y no se cuenta con un registro adecuado sobre su prevalencia (1). La prevalencia de hipertensión arterial se ha estimado en $14.1 \%$ en población de 20-69 años que vive en el área urbana, una de las más bajas respecto de otros países latinoamericanos, pero supera 50\% entre sujetos de 60-69 años, una cifra similar a la de los Estados Unidos. De las hospitalizaciones debidas a trastornos cardiovasculares, se ha descrito que hasta $40 \%$ se atribuye a FC (4).

De acuerdo con referentes internacionales, en países desarrollados, el gasto ocasionado por la FC representa entre 1 y $2 \%$ del costo total en salud y aproximadamente dos tercios de los gastos asociados con FC se atribuyen a eventos hospitalarios relacionados, no solo con descompensación de la FC, sino además con las comorbilidades que suelen acompañar a la FC (5). En Latinoamérica son escasas las publicaciones sobre evaluaciones económicas en FC, Giorgi y cols. realizaron un descripción de los costos asociados con la atención hospitalaria de pacientes adultos con FC aguda, en la cual se identificó que el costo entre aquellos pacientes con función sistólica disminuida (fracción de eyección del ventrículo izquierdo menor a 50\%) resultó $27 \%$ superior al costo asociado con la atención de pacientes con la función sistólica conservada (6). Sobre la determinación de costos asociados con la atención de la FC en Colombia, son también pocas las publicaciones que hacen referencia a ello. Recientemente, dentro del marco de un análisis de costo-efectividad de agentes betabloqueadores en el tratamiento de la FC, se estimó el costo promedio del evento hospitalario en 3.260.772 pesos colombianos (COP) (D.E. 113.117 COP) de 2009 (7).

Frente a la incertidumbre, en el escenario colombiano, de la costo-efectividad y la costo-utilidad de múltiples intervenciones farmacológicas y no farmacológicas en FC, es necesario determinar una línea de base que defina los costos directos asociados con el tratamiento de esta entidad, tanto en su estado compensado que se aborda de manera ambulatoria, como en su estado de descompensación que demanda atención hospitalaria. Este estudio tiene por objetivo realizar una aproximación a la determinación de los costos directos de la FC en el país, a través de la evaluación de los costos asociados con el cuidado de la FC en una muestra de pacientes atendidos en dos instituciones prestadoras de servicios de salud de alto nivel de complejidad de Bogotá. Los resultados podrían constituir un aporte para la definición de una línea de base que sirva como insumo para la evaluación del impacto económico de intervenciones sanitarias dirigidas a este grupo de pacientes.

\section{Métodos}

Se condujo un estudio de costos bajo la perspectiva del tercer pagador. En el caso de la atención ambulatoria de la FC compensada, la identificación de los eventos generadores de costos se realizó mediante la revisión de historias clínicas de una cohorte de pacientes adultos atendidos durante 2011 en el servicio de consulta externa especializada dirigida a pacientes con FC de una institución hospitalaria de tercer nivel de complejidad de Bogotá. De la historia clínica de cada paciente se obtuvo información sobre la edad, el sexo, la frecuencia de consulta de seguimiento, la frecuencia de solicitud de interconsultas y de estudios paraclínicos (laboratorios clínicos y exámenes diagnósticos) y la prescripción de medicamentos (denominación, presentación farmacéutica, dosis, periodicidad).

Los costos de interconsultas y de estudios paraclínicos se determinaron según los valores definidos en el Acuerdo 256 de 2001 (8), con adición de 30\% para aproximar a los valores de los acuerdos de contratación entre prestadores y aseguradores vigentes para 2011; tal adición se incorporó de acuerdo con lo definido por el consenso del grupo de- 
sarrollador de las más recientes guías de atención integral de la Pontificia Universidad Javeriana en conjunto con prestadores de servicios de salud (9) y que, se consideró un referente local legítimo para la estimación de costos de los recursos cuyo consumo se identificó en el escenario de la atención ambulatoria de la FC. Los costos de la medicación se determinaron a partir de las cifras reportadas en el canal de compras del registro SISMED correspondiente al periodo comprendido entre enero y diciembre de 2011 (10). Se seleccionaron cinco registros de cada medicamento y se determinó el costo promedio por unidad básica de medida (mg, UI, Lt, mcg) de cada medicamento.

En el caso de las hospitalizaciones debidas a descompensación de la FC, la identificación de eventos generadores de costos se realizó mediante la revisión de listados consolidados y facturas de una cohorte de pacientes adultos atendidos entre 2009 y 2010 en dos instituciones hospitalarias de tercer nivel de complejidad de Bogotá. Se consideraron elegibles los registros de pacientes con diagnóstico principal de egreso de FC descompensada y edad de 18 años o más. Se obtuvo además información sobre la duración de estancia. Se obtuvo también la factura generada por la institución hospitalaria al ente asegurador $\mathrm{y}$, a partir de ésta, se determinaron los costos directos por la atención del evento hospitalario.

De acuerdo con la información disponible en las facturas, los costos se agruparon según el centro de costos que los generó (estancia, honorarios médicos, laboratorios, imágenes diagnósticas, medicamentos, insumos y procedimientos quirúrgicos). Los valores reportados en cada factura fueron traídos a valor presente en COP de 2011, para lo cual se usó el factor deflactor basado en el valor reportado de canasta familiar conocido comúnmente por sus nombre en inglés como "GDP -gross domestic product", por el Banco Mundial para Colombia en los años 2009, 2010 y 2011 (11).

Los resultados de este proceso de costeo se presentan resumidos por medidas de tendencia central y de dispersión. Dado que los costos hospitalarios presentaron gran heterogeneidad relacionada con valores extremos, tanto bajos como altos, y que dichos valores extremos podrían reflejar el efecto del tipo de contratación pactado entre aseguradoras y prestadores y la presencia de eventos clínicos infrecuentes -potencialmente más costosos-, se consideró que una medida más adecuada para resumir los costos hospitalarios sería la media recortada al 10\% (5\% de los valores mínimos y $5 \%$ de los valores máximos). El análisis fue realizado utilizando el programa STATA® 11.

\section{Resultados \\ Atención ambulatoria de FC compensada}

Se revisaron las historias clínicas de 158 pacientes atendidos en 2011 en el servicio de consulta externa especializada dirigida a pacientes con FC de una institución hospitalaria de tercer nivel de complejidad de Bogotá, que correspondieron a 502 consultas. El promedio de edad de estos pacientes fue de 62 años (D.E. 15 años) y $63.9 \%$ eran hombres. Se identificó que, en promedio, este perfil de pacientes asistió a consulta de seguimiento tres veces (D.E. 1,9) en el año.

De acuerdo con los rubros costeados y los valores obtenidos, se encontró que el costo mensual promedio del esquema de tratamiento ambulatorio de FC de estos pacientes fue 304.318 COP (D.E. 760.876), con una mediana de 45.280 COP (RIC 25.539 - 109.715). Se encontró que la fuente de consumo de recursos que representó mayor proporción de costos fue la de medicamentos, con 55.2\%, tal como se observa en la Tabla 1.

En relación con la prescripción farmacológica, se encontró que los diuréticos (espironolactona en $66.9 \%$ de las consultas, furosemida en $38.1 \%$ e hidroclorotiazida en $2 \%$ ), los betabloqueadores (67.7\%) y los inhibidores de la enzima convertidora de angiotensina / antagonistas del receptor de angiotensina II $(65,1 \%)$ fueron los medicamentos prescritos con mayor frecuencia. Las especialidades médicas a las cuales se generó interconsulta con mayor frecuencia fueron electrofisiología (4\%) y neumología (2.2\%). Los estudios paraclínicos más solicitados correspondieron a tiempo de protrombina (en algunos casos con INR), parámetros séricos de función renal, electrolitos, electrocardiograma y ecocardiograma transtorácico.

\section{Atención hospitalaria de FC descompensada}

Se revisaron los registros, en los listados consolidados debidos a descompensación de FC suministrados por las dos instituciones hospitalarias, de 352 y 1074 eventos hospitalarios respectivamente, para un total de 1426 hospitalizaciones sucedidas entre 2009 y 2010. La duración promedio de la estancia hospitalaria fue de ocho días (D.E. 11), con una mediana de seis días (RIC 3-9), mientras que 3.7\% de los eventos se registró una estancia superior a 30 días.

Los rubros costeados y los valores obtenidos se presentan en resumen en la Tabla 2. Bajo la consideración sobre uso de la media recortada a $10 \%$, se identificó que el costo promedio del evento hospitalario en este grupo de pacientes (90\% central de la distribución) fue de 6.427.887 COP (D.E. 9.663.176).

Se encontró que el rubro que representó mayor proporción del costo total fue el de estancia hospitalaria, con $29.1 \%$ (D.E. 16.1) mientras que el de menor proporción fue el de honorarios médicos, responsable, en promedio, de $13.7 \%$ (D.E. 12.3) del costo, como se presenta en la Tabla 3.

Tabla 1. Distribución porcentual del costo de atención ambulatoria de pacientes en la Clínica de Falla Cardiaca de una institución hospitalaria de tercer nivel de complejidad de Bogotá.

\begin{tabular}{|l|c|c|}
\hline Rubro & Porcentaje promedio & D.E. \\
\hline Laboratorios & 18.8 & 19.5 \\
Exámenes & 22.4 & 28.7 \\
Medicamentos & 55.2 & 31.2 \\
Interconsultas & 3.7 & 6.3 \\
\hline
\end{tabular}


Tabla 2. Costos de atención hospitalaria de pacientes con descompensación de falla cardiaca en dos hospitales de Bogotá.

\begin{tabular}{|l|c|c|}
\hline \multirow{2}{*}{ Estimación } & \multicolumn{2}{|c|}{ Costo (pesos colombianos 2011) } \\
\cline { 2 - 3 } & $\begin{array}{c}\text { Total } \\
\mathbf{n = 1 4 2 6}\end{array}$ & $\begin{array}{c}\mathbf{9 0 \%} \text { Central } \\
\mathbf{n}=\mathbf{1 1 4 6}\end{array}$ \\
\hline Mínimo & $\$ 5.305$ & $\$ 137.920$ \\
Máximo & $\$ 265.772 .641$ & $\$ 59.731 .298$ \\
P25 & $\$ 1.505 .962$ & $\$ 1.698 .283$ \\
Mediana & $\$ 3.171 .020$ & $\$ 3.171 .020$ \\
P75 & $\$ 6.804 .665$ & $\$ 6.232 .268$ \\
Promedio & $\$ 10.400 .214$ & $\$ 6.427 .887$ \\
D.E. & $\$ 22.552 .954$ & $\$ 9.663 .176$ \\
\hline
\end{tabular}

\section{Discusión}

El presente estudio muestra que los costos ambulatorios, y especialmente los hospitalarios asociados con la FC en Colombia son sustanciales, y que la fuente principal de los costos difiere dependiendo de si el manejo es hospitalario (estancia) o ambulatorio (medicamentos).

De acuerdo con hallazgos en publicaciones tanto locales como internacionales, son escasos los estudios que se han desarrollado en torno a los costos asociados con la atención de la FC en Colombia. Los resultados de esta investigación, en Colombia, ofrecen una aproximación a los costos médicos directos derivados del cuidado de esta condición a partir de fuentes primarias, con una población que expone el escenario de varios pagadores y por ende varios acuerdos de contratación, lo cual conduce a que las cifras obtenidas reflejen tanto el escenario clínico como el de consumo de recursos de la realidad de las instituciones participantes. Este perfil de información puede servir como insumo para futuras evaluaciones económicas de diversas intervenciones dirigidas a los pacientes con FC en Colombia e incluso, como herramienta para planificación e implementación de servicios de salud con impacto tanto económico como en calidad de vida de los pacientes (5). Adicionalmente, este estudio tuvo en cuenta, además de los costos hospitalarios, el escenario del consumo de recursos y el costo la atención ambulatoria de la FC, hasta ahora poco explorado en Colombia.

La duración promedio de la estancia hospitalaria entre los pacientes de este estudio (ocho días) resultó algo mayor que la que se ha identificado en grandes cohortes descritas en literatura internacional. En un registro norteamericano, obtenido a partir de datos almacenados y disponibles en bases de datos estandarizadas (Medicare Enrollment Database de los servicios del Medicare), se identificó que la estancia promedio en eventos hospitalarios fue de 6.8 días (D.E. 11), con un rango de 3.5 a 11.9 días, entre 1315845 admisiones por FC, de pacientes de 65 años y mayores, entre 2004 y 2006 en 4209 hospitales; en el contexto de este mismo registro, el promedio de estancia hospitalaria por FC resultó cercano al de condiciones como infarto agudo del miocardio y neumonía cuya estancia promedio
Tabla 3. Distribución porcentual del costo de hospitalización de pacientes con falla cardiaca en dos hospitales de Bogotá.

\begin{tabular}{|l|c|c|}
\hline Rubro & $\begin{array}{c}\text { Porcentaje promedio } \\
\text { de 90\% central }\end{array}$ & D.E. \\
\hline Estancia & 29.1 & 16.1 \\
Honorarios & 13.7 & 12.3 \\
Medicamentos & 27.2 & 15.8 \\
Laboratorios & 14.1 & 10 \\
Otros & 20.8 & 13 \\
\hline
\end{tabular}

fue de 7.1 (D.E. 1.5 para infarto agudo del miocardio y 1.2 para neumonía) (12).

Esta cifra de duración promedio de estancia hospitalaria coincide con los hallazgos reportados por Bueno y cols. - a partir de datos obtenidos también de los registros de servicios del Medicare - entre 844327 hospitalizaciones por FC ocurridas entre 2007 y 2008, en pacientes con una edad promedio de 80.4 años (D.E. 8.2), cuya estancia promedio fue de 6.32 días (D.E. 5.0), con una mediana de cinco días (P25 4-P75 7) (13). Sin embargo, en el presente estudio no se exploraron otras variables, como las comorbilidades o las complicaciones ocurridas durante la hospitalización, u otros desenlaces, como la mortalidad intrahospitalaria, que pudieran exponer el panorama clínico que condujo a una mayor estancia respecto de los referentes internacionales.

El costo promedio por evento hospitalario determinado en este estudio (6.427.887 COP) contrasta con el descrito recientemente por Romero y cols. (7) dentro del marco de un análisis de costo-efectividad de una intervención farmacológica en FC; entre 513 pacientes se encontró un valor promedio del evento hospitalario de 3.260.772 COP de 2009 (D.E. 113.017 COP), que resulta casi la mitad del valor para 2011 identificado en este estudio, lo cual puede deberse a que el estudio de Romero y cols. incluyó también pacientes con diagnóstico asociado de hipertensión arterial, cuyo consumo de recursos podría diferir del propio para FC sin tal asociación, mientras que para el caso del presente estudio, se tuvieron en cuenta casos con diagnóstico de FC sin restricción a la asociación con hipertensión arterial.

De acuerdo con la información reportada por el Sistema Integral de Información de la Protección Social (SISPRO) del Ministerio de Salud y Protección Social de Colombia, el promedio de estancia en hospitalizaciones debidas a FC fue de 9.21 días en 2009, 7.8 días en 2010 y 10.72 en 2011 , cifras que, resultaron superiores a las del promedio de estancia en hospitalización por cualquier causa: 6.96 en 2009, 3.64 en 2010 y 5.69 en 2011 (14). La estancia promedio en hospitalización por FC, correspondiente a la muestra del presente estudio, resultó cercana, y en algunos casos inferior, a los promedios nacionales reportados por SISPRO tanto para el régimen contributivo como para el régimen subsidiado: 9.49 y 8.97, respectivamente, en 2009; 7.57 y 7.90 en 2010 ; y 11.5 y 9.36 en 2011 (14). 
En relación con las limitaciones de este estudio, primero, se debe considerar que no se evaluó el impacto de la presencia de condiciones médicas coexistentes, tanto comorbilidades previas como complicaciones durante la hospitalización, lo cual hubiese podido explicar por qué el consumo de recursos (como estancia hospitalaria) y los costos resultaron mayores que los descritos en otras cohortes. Sin embargo, la FC es un problema multicausal asociado casi siempre con diferentes comorbilidades, lo cual hace poco razonable, y un ejercicio subjetivo, tratar de atribuir consumos de recursos diferenciales entre FC y otras condiciones coexistentes. En segundo lugar se tiene un escenario de limitada generalizabilidad de los resultados para población fuera del régimen contributivo y de la medicina prepagada, que corresponde al perfil de población atendida en las instituciones participantes.

Es recomendable en nuevos estudios en esta área, incorporar población atendida en prestadores de otros regímenes de afiliación y su caracterización demográfica, además de la medición de otras variables (funciones sistólica y diastólica, clase funcional, comorbilidades, tipo de cardiopatía, presentación de la descompensación) y desenlaces que ayudarían a complementar la información sobre el panorama de la FC en Colombia y a obtener referentes para comparar con escenarios internacionales y a través del tiempo, como la mortalidad intrahospitalaria, la mortalidad a 30 días y la proporción de reingresos. Incluso, valdría la pena evaluar en una muestra de pacientes suficiente y representativa, la asociación entre la especialidad tratante y los desenlaces clínicos relevantes y los costos asociados, ajustando por atributos como la severidad de la descompensación de la FC y las comorbilidades presentes.

\section{Declaración de fuentes de financiación y posibles conflictos de interés}

Los autores manifiestan que, durante el desarrollo de este estudio y la posterior elaboración de este manuscrito, no tuvieron vinculación alguna con entidades o actividades que representen algún conflicto de interés en relación con el diseño, ejecución y difusión de esta investigación.

Así mismo, ninguno de los autores fue partícipe de actividades financieras relacionadas con compañías farmacéuticas o con otras empresas que pudieran constituir conflicto de interés alguno.

Fuentes de apoyo. Este trabajo se condujo dentro del marco de una investigación desarrollada con el respaldo financiero de Colciencias y la cofinanciación dada por la Fundación Cardioinfantil - Instituto de Cardiología, la Pontificia Universidad Javeriana y la Universidad de McGill.

\section{Reconocimientos y agradecimientos}

Los autores declaran su reconocimiento a Colciencias por la financiación a través de contrato derivado de la convocatoria 519-2010; a la Fundación Cardioinfantil - Instituto de Cardiología y a la Pontificia Universidad Javeriana por el respaldo financiero para la realización de este estudio; así mismo, manifiestan su agradecimiento a María Sacramento Esguerra, auxiliar de enfermería, por su trabajo en la recolección y digitación de la información sobre historias clínicas y facturas y a las directivas de la Fundación Cardioinfantil - Instituto de Cardiología y del Hospital Universitario San Ignacio por permitir el acceso a los registros clínicos y de facturación de los pacientes con FC atendidos en estas instituciones.

\section{Referencias}

1. Accini J, Gómez E, Atehortúa L, Gómez J, Castillo G, Ortiz G, et al. Guías de tratamiento de la falla cardíaca aguda. Rev Col Cardiología 2011; 18(Supl. 2): $165-99$

2. Hernández-Leiva E. Epidemiología del síndrome coronario agudo y la insuficiencia cardiaca en Latinoamérica. Rev Esp Cardiol 2011;64(Supl. 2): 34-43.

3. Senior J, Saldarriaga C, Rendón J. Descripción clínico-epidemiológica de los pacientes con falla cardiaca aguda que consultan al servicio de urgencias. Acta Med Colomb 2011; 36: 125-9.

4. Cubillos-Garzón L, Casas J, Morillo C, Bautista L. Congestive heart failure in Latin America: The next epidemic. Am Heart J 2004; 147: 412-7.

5. Ferrante D. Costos de la insuficiencia cardíaca. Rev Argent Cardiol 2008; 76(1): 6-7.

6. Giorgi M, Ahuad R, Voos M, Borracci R, Farrás H, Insúa J. Análisis de costos hospitalarios de la insuficiencia cardíaca con función sistólica conservada versus deteriorada. Rev Argent Cardiol 2008; 76(1): 20-6.

7. Romero M,Arango C. Análisis de costo efectividad del uso de metoprolol succinato en el tratamiento de la hipertensión arterial y la falla cardiaca en Colombia. Rev Col Cardiol 2012; 19(4): 160-8.

8. Consejo Directivo del Instituto de Seguros S. Acuerdo No. 256 de 2001. POR EL CUAL SE APRUEBA EL "MANUAL DE TARIFAS" DE LA ENTIDAD PROMOTORA DE SALUD DEL SEGURO SOCIAL “EPS-ISS”. 2001. p. 1-211.

9. Chicaíza-Becerra L, García-Molina M, Oviedo S, Gómez-Marín J, Gómez P. Costo efectividad de diferentes estrategias diagnósticas para detección de toxoplasmosis congénita en el recién nacido. 2012; 37:1-33. Disponibe en: http://www.fce.unal.edu.co/media/files/doc_escuela_economa_n37_chicaza garcia.pdf. Disponible en: http://www.fce.unal.edu.co/publicaciones/ index.php?option=com_content $\&$ view=article $\&$ id=178:37-costo-efectividad-dediferentes-estrategias-diagnosticas-para-deteccion-de-toxoplasmosis-congenita-enel-recien-nacido\&catid=42: documentos-fce-cid-escuela-de-economia\&Itemid=56.

10. Ministerio de Salud y Protección Social. Listado de precios promedio y unidades en la cadena de comercialización de medicamentos - Enero a diciembre de 2011. 2012

11. The World Bank. 2012 [updated November 16-2012] [Nov-2012]. Disponible en: http://data.worldbank.org/indicator/NY.GDP.MKTP.CD.

12. Drye E, Normand S, Wang Y, Ross J, Schreiner G, Han L, et al. Comparison of Hospital Risk-Standardized Mortality Rates Calculated by Using In-Hospital and 30-Day Models: An Observational Study With Implications for Hospital Profiling. Ann Intern Med 2012; 156(1): 19-26.

13. Bueno H, Ross J, Wang Y, Chen J, Vidán MT, Norman SL, et al. Trends in length of stay and short-term outcomes among Medicare patients hospitalized for heart failure, 1993 - 2006. JAMA 2010; 303(21): 2141-7.

14. SISPRO. Bodega de datos de SISPRO (RIPS) - Ministerio de Salud y Protección Social. Reporte: prestaciones de servicios de salud reportados por las entidades administradoras de planes de beneficio para los años 2009, 2010 y 2011, según resolución 3374/00. Actualización a 31-01-2013. Consultado: 11-03-13. 